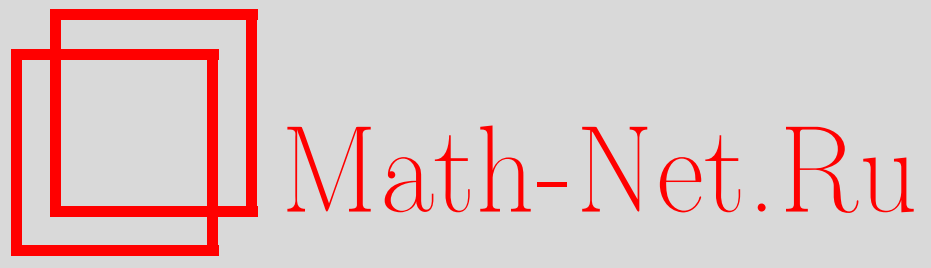

Обцероссийский математический портал

Ю. А. Коняев, Асимптотическое разложение определителя возмущенной матрицы, Матем. заметки, 2004, том 76, вы- 
пуск 1, 149-151

DOI: https://doi.org/10.4213/mzm567

Использование Общероссийского математического портала Math-Net.Ru подразумевает, что вы прочитали и согласны с пользовательским соглашением http://www.mathnet.ru/rus/agreement

Параметры загрузки:

IP : 54.174 .149 .18

26 апреля 2023 г., 13:43:36 


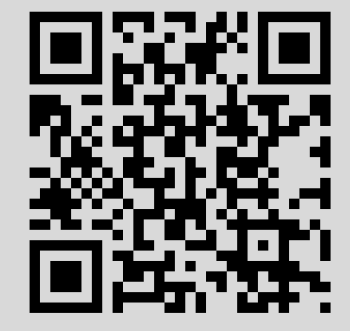




\section{АСИМПТОТИЧЕСКОЕ РАЗЛОЖЕНИЕ ОПРЕДЕЛИТЕЛЯ ВОЗМУЩЕННОЙ МАТРИЦЫ}

\section{Ю. А. Коняев}

Изложен достаточно простой и конструктивный метод асимптотического разложения возмущенной матрицы, что дополняет известные ранее результаты классической теории возмущений [1]-[3].

Предложенный алгоритм является развитием идей работы [4]. Рассмотрим определитель возмущенной квадратной $n \times n$ матрицы

$$
A(\varepsilon)=\sum_{0}^{\infty} A_{k} \varepsilon^{k}, \quad|\varepsilon|<1,
$$

считая известными собственные значения $\left\{\lambda_{0 j}\right\}_{1}^{n}$ и собственные векторы $\left\{s_{0 j}\right\}_{1}^{n}$ невозмущенной $(\varepsilon=0)$ матрицы $A_{0}$, при этом $\operatorname{det} A_{0}=\prod_{1}^{n} \lambda_{0 j}=D_{0}$.

При известном разложении спектра

$$
\lambda_{j}(\varepsilon)=\sum_{0}^{\infty} \lambda_{k j} \varepsilon^{k}, \quad j=1, \ldots, n
$$


возмушенной матрицы $A(\varepsilon)$ решение поставленной задачи может быть представлено в виде

$$
\operatorname{det} A(\varepsilon)=\prod_{1}^{n} \lambda_{j}(\varepsilon)=\sum_{0}^{\infty} D_{k} \varepsilon^{k}
$$

Как известно [1], [2], [4], построение разложений (1) является далеко не тривиальной задачей и его структура заметно усложняется при возмущении матрицы $A_{0}$ произвольной жордановой структуры, что в общем случае может привести к разложению точек спектра $\lambda_{j}(\varepsilon)$ по некоторым дробньм степеням малого параметра.

В работе [4] изложен, отличный от ранее известного [1], [2], конструктивный метод одновременного построения всех собственных значений $\lambda_{j}(\varepsilon)$ и собственных векторов $s_{j}(\varepsilon), j=1, \ldots, n, \mathrm{c}$ учетом структуры предельой $(\varepsilon=0)$ матрицы $A_{0}$.

В данной заметке (в развитие идей работы [4]) предложен простой алгоритм решения поставленной задачи (2) для случая предельной матрицы $A_{0}$ произвольной жордановой структуры (при наличии не более, чем одного нулевого собственного значения), позволяющий записать асимптотическое представление (2) по целым степеням малого параметра $\varepsilon$ независимо от кратности ненулевых точек спектра $\left\{\lambda_{0 j}\right\}_{1}^{n}$ матрицы $A_{0}$.

Если $J_{0}=S_{0}^{-1} A_{0} S_{0}=\Lambda_{0}+W ; \Lambda_{0}=\operatorname{diag}\left\{\lambda_{01}, \ldots, \lambda_{0 n}\right\}, \operatorname{det} S_{0} \neq 0$ (где $W$ - некоторая нильпотентная матрица с достаточно малой нормой $\|W\|<1$ ), то при сделанных предположениях всегда можно подобрать невырожденную матрицу $R_{0}$ такую, что $P_{0}=R_{0} J_{0}$ будет иметь только простые (некратные) собственные значения, допуская ее приведение после невырожденного преобразования $U_{0}$ к диагональной матрице $N_{0}=U_{0}^{-1} P_{0} U_{0}=\operatorname{diag}\left\{\nu_{01}, \ldots, \nu_{0}\right\}$, что позволяет воспользоваться теоремой 1 работы [4].

Действительно, введя для произвольной квадратной матрицы $A$ обозначения

$$
A=\left\{a_{j k}\right\}_{1}^{n}, \quad \bar{A}=\operatorname{diag}\left\{a_{11}, \ldots, a_{n n}\right\}, \quad \overline{\bar{A}}=A-\bar{A},
$$

рассмотрим следующие матрицы:

$$
B(\varepsilon)=S_{0}^{-1} A(\varepsilon) S_{0}=J_{0}+\sum_{1}^{\infty} B_{k} \varepsilon^{k}, \quad P(\varepsilon)=U_{0}^{-1} R_{0} B(\varepsilon) U_{0}=N_{0}+\sum_{1}^{\infty} P_{k} \varepsilon^{k},
$$

для которых имеет место соотношение $\operatorname{det} P(\varepsilon)=\operatorname{det} R_{0} \operatorname{det} A(\varepsilon)$; тогда решение задачи (2) определяется спектром $\nu_{j}(\varepsilon)$ матрицы $P(\varepsilon)$ и сводится (с учетом резултатов работы [4]) к решению матричного уравнения

$$
P(\varepsilon) H(\varepsilon)=H(\varepsilon) N(\varepsilon),
$$

которое имеет единственное решение

$$
H(\varepsilon)=E+\sum_{1}^{\infty} \overline{\bar{H}}_{k} \varepsilon^{k}, \quad N(\varepsilon)=\sum_{0}^{\infty} N_{k} \varepsilon^{k}=\operatorname{diag}\left\{\nu_{1}(\varepsilon), \ldots, \nu_{n}(\varepsilon)\right\}
$$

в классе диагональных $N_{k}=\operatorname{diag}\left\{\nu_{k 1}, \ldots, \nu_{k n}\right\}, k \geqslant 0$, и бездиагональных $\overline{\bar{H}}_{k}$ матриц.

Приравнивая в уравнении (3) коэффициенты при одинаковых степенях $\varepsilon$, получим простые алгебраические уравнения

где $F_{1}=P_{1}$,

$$
N_{0} \overline{\bar{H}}_{k}-\overline{\bar{H}}_{k} N_{0}=N_{k}-F_{k}, \quad k \geqslant 1,
$$

$$
F_{k}=P_{k}+\sum_{j=1}^{k-1}\left(P_{j} \overline{\bar{H}}_{k-j}-\overline{\bar{H}}_{k-j} N_{j}\right)=\left\{f_{j g}\right\}_{k} .
$$

Из соотношения (4) последовательно и однозначно определяются все диагональные $N_{k}$ и бездиагональные матрицы $\overline{\bar{H}}_{k}, k \geqslant 1$, при этом

$$
N_{k}=\bar{F}_{k}, \quad \overline{\bar{H}}_{k}=\left\{h_{j q}\right\}_{k}, \quad\left(h_{j q}\right)_{k}=\left(\lambda_{0 j}-\lambda_{0 k}\right)^{-1}\left(f_{j q}\right)_{k}, \quad j \neq q, \quad j, q=1, \ldots, n, \quad k \geqslant 1 .
$$

Таким образом, имеет место утверждение. 
ТЕОРемА. При наличии у невозмущенной матрицы $A_{0}$ с произвольной жсордановой структурой не более одного нулевого собственного значения имеет место асимптотическое разлохсение определителя возмущенной матрииы $A(\varepsilon)$ :

$$
\operatorname{det} A(\varepsilon)=\operatorname{det}^{-1} R_{0} \operatorname{det} P(\varepsilon)=\operatorname{det}^{-1} R_{0} \prod_{1}^{n} \nu_{j}(\varepsilon)=\sum_{0}^{\infty} D_{k} \varepsilon^{k},
$$

определяемое по указанному выше алгоритму.

Случай, когда матрица $A_{0}$ эквивалентна матрице, содержащей жорданову клетку с нулевым кратным собственным значением:

$$
J_{0}=S_{0}^{-1} A_{0} S_{0}=\operatorname{diag}\left\{J_{01}, W_{02}\right\},
$$

где

$$
J_{01}=\Lambda_{01}+W_{01}, \quad \Lambda_{01}=\operatorname{diag}\left\{\lambda_{01}, \ldots, \lambda_{0 q}\right\}, \quad 1 \leqslant q<n,
$$

$W_{01}, W_{02}$ - некоторые нильпотентные матрицы, может быть исследован методами работы [4] с помощью обобщенного срезающего образования, приводящему, вообще говоря, к разложению $\operatorname{det} A(\varepsilon)(2)$ по некоторым дробньм степеням малого параметра.

\section{СПИСОК ЦИТИРОВАННОЙ ЛИТЕРАТУРЫ}

1. Като Т. Теория возмущений линейных операторов. М.: Мир, 1972. 2. Ланкастер П. Теория матриц. М.: Наука, 1978. 3. Воеводин В. В. Вычислительные основы линейной алгебры. М.: Наука, 1977. 4. Коняев Ю. А. // Матем. сб. 1993. Т. 184. № 12. С. 133-144.

Российский университет Дружбы народов 